\title{
Minimum MSE Weights of Adjusted Summary Estimator of Risk Difference in Multi-Center Studies
}

\author{
Chukiat Viwatwongkasem $^{1 *}$, Jirawan Jitthavech ${ }^{2}$, Dankmar Böhning ${ }^{3}$, Vichit Lorchirachoonkul ${ }^{2}$ \\ ${ }^{1}$ Department of Biostatistics, Faculty of Public Health, Mahidol University, Bangkok, Thailand \\ ${ }^{2}$ School of Applied Statistics, National Institute of Development Administration, Bangkok, Thailand \\ ${ }^{3}$ Applied Statistics, School of Biological Sciences, University of Reading, Reading, UK \\ Email: "phcvw@mahidol.ac.th
}

Received October 14, 2011; revised November 18, 2011; accepted November 30, 2011

\begin{abstract}
The simple adjusted estimator of risk difference in each center is easy constructed by adding a value $c$ on the number of successes and on the number of failures in each arm of the proportion estimator. Assessing a treatment effect in multi-center studies, we propose minimum MSE (mean square error) weights of an adjusted summary estimate of risk difference under the assumption of a constant of common risk difference over all centers. To evaluate the performance of the proposed weights, we compare not only in terms of estimation based on bias, variance, and MSE with two other conventional weights, such as the Cochran-Mantel-Haenszel weights and the inverse variance (weighted least square) weights, but also we compare the potential tests based on the type I error probability and the power of test in a variety of situations. The results illustrate that the proposed weights in terms of point estimation and hypothesis testing perform well and should be recommended to use as an alternative choice. Finally, two applications are illustrated for the practical use.
\end{abstract}

Keywords: Minimum MSE Weights; Optimal Weights; Cochran-Mantel-Haenszel Weights; Inverse Variance Weights; Multi-Center Studies; Risk Difference

\section{Introduction}

It is widely known that the conventional proportion estimator, $\hat{p}=X / n$, is a maximum likelihood estimator (MLE) and an uniformly minimum variance unbiased estimator (UMVUE) for the binomial parameter $p$ where the binomial random variable $X$ is the number of successes out of the number of patients $n$. However, Agresti and Coull [1], Agresti and Caffo [2], Ghosh [3], and Newcombe [4,5] highlighted the point that $\hat{p}$ might not be a good choice for $p$ when the assumption of $n \hat{p} \geq 5$ and $n(1-\hat{p}) \geq 5$ was violated; this violation often occurs when the sample size $n$ is small, or the estimated probability $\hat{p}$ is close to 0 or 1 (close to the boundaries of parameter space), leading to the problem of the zero estimate of the variance of $\hat{p}$. The estimated variance of $\hat{p}$, provided by $\hat{V}(\hat{p})=\hat{p}(1-\hat{p}) / n$, is zero in the occurrence of any case: $X=0$ or $X=n$. Böhning and Viwatwongkasem [6] proposed the simple adjusted proportion estimator by adding a value $c$ on the number of successes and the number of failures; conesquently, $\hat{p}_{c}=(X+c) /(n+2 c)$ is their proposed estimate of $p$ with the non-zero variance estimate

\footnotetext{
*Corresponding author.
}

$\hat{V}\left(\hat{p}_{c}\right)=n \hat{p}_{c}\left(1-\hat{p}_{c}\right) /(n+2 c)^{2}$. They concluded that the estimator $(X+1) /(n+2)$ minimizes the Bayes risk (the average MSE of $\left.\hat{p}_{c}\right)$ in the class of all estimators of the form $(X+c) /(n+2 c)$ with respect to uniform prior on $[0,1]$ and Euclidean loss function; furthermore, the estimator $(X+1) /(n+2)$ has smaller MSE than $X / n$ in the approximate interval $[0.15,0.85]$ of $p$. For another argumentation in the Bayesian approach, Casella and Berger [7] showed that $(X+\alpha) /(n+\alpha+\beta)$ is a Bayes estimator of $p$ under the conditional binomial sampling $X \mid p \sim \operatorname{binomial}(n, p)$ and the prior beta distribution $p \sim \operatorname{beta}(\alpha, \beta)$. Note that in case of $\alpha=\beta=1$ the beta distribution has a special case as the uniform distribution over [0,1]. Consequently, the estimator $(X+c) /(n+2 c)$ derived from the Bayesian approach and the Bayes risk approach under the above mentioned criteria provides the same result at $c=1$.

With the idea of $\hat{p}_{c}=(X+c) /(n+2 c)$, the extension leads to $\hat{\theta}_{c}=\hat{p}_{c 1}-\hat{p}_{c 2}$, the adjusted risk difference estimator between two independent binomial proportions, for estimating a common risk difference $\theta$ where $\hat{p}_{c 1}=\left(X_{1}+c_{1}\right) /\left(n_{1}+2 c_{1}\right)$ and $\hat{p}_{c 2}=\left(X_{2}+c_{2}\right) /\left(n_{2}+2 c_{2}\right)$ are proportion estimators for treatment and control arms. In a multi-center study of size $k$, the parameter of in- 
terest is also a common risk difference $\theta$ that is assumed to be a constant across centers. We concern about a combination of several adjusted risk difference estimators $\hat{\theta}_{c j}=\hat{p}_{c 1 j}-\hat{p}_{c 2 j}$ from the $j^{\text {th }}$ center $(j=1,2, \cdots, k)$ into the adjusted summary estimator of risk difference of the form $\hat{\theta}_{c w}=\sum_{j=1}^{k} f_{c j} \hat{\theta}_{c j}$ where $f_{c j}$ are the weights subject to the condition that $\sum_{j=1}^{k} f_{c j}=1$. In this study, we would propose the optimal weights $f_{c j}$ as an alternative choice based on minimizing the MSE of $\hat{\theta}_{c w}$ in Section 2, then state the well-known candidates such as the Cochran-Mantel-Haenszel (CMH) weights and the inverse variance (INV) weights in Section 3. A simulation plan for comparing the performance among weights in terms of estimation and hypothesis testing is presented in Section 4. The results of the comparison among the potential estimators based on bias, variance, and MSE and also the evaluations among tests related the mentioned weights through the type I error probability and the power criteria lie on Section 5. Some numerical examples are applied in Section 6. Finally, conclusion and discussion are presented in Section 7.

\section{Deriving Minimum MSE Weights of Adjusted Summary Estimator}

Under the assumption of a constant of common risk difference $\theta$ across $k$ centers, we combine several adjusted risk difference estimators $\hat{\theta}_{c j}=\hat{p}_{c 1 j}-\hat{p}_{c 2 j}$ in which $\hat{p}_{c 1 j}=\left(X_{1 j}+c_{1}\right) /\left(n_{1 j}+2 c_{1}\right)$ and $\hat{p}_{c 2 j}=\left(X_{2 j}+c_{2}\right) /\left(n_{2 j}+2 c_{2}\right)$ from the $j^{\text {th }}$ center $(j=1,2, \cdots, k)$ arrive at an adjusted summary estimator of risk difference of the form $\hat{\theta}_{c w}=\sum_{j=1}^{k} f_{c j} \hat{\theta}_{c j}$ where $f_{c j}$ are non-random weights subject to the constraint that $\sum_{j=1}^{k} f_{c j}=1$. Please observe that for a single center $(k=1)$ the adjusted summary estimator $\hat{\theta}_{c w}=\sum_{j=1}^{k} f_{c j} \hat{\theta}_{c j}$ subject to $\sum_{j=1}^{k} f_{c j}=1$ is a shrinkage estimator of a simple adjusted estimator $\hat{\theta}_{c}=\hat{p}_{c 1}-\hat{p}_{c 2}$. Our minimum MSE weights $f_{c j}$ of the adjusted summary estimator $\hat{\theta}_{c w}$ were derived by following Lagrange's method [8] under the assumption of a constant of common risk difference over all centers with the pooling point estimator to estimate $\theta$. Lui and Chang [9] proposed the optimal weights proportional to the reciprocal of the variance with the Mantel-Haenszel point estimator under the assumption of noncompliance. It was observed that both of optimal weights provided the different formulae because of different assumptions even though they were derived from the same method of Lagrange. Now, we wish to present the proposed weights minimizing the MSE of $\hat{\theta}_{c w}$ as follows:

$$
Q=\operatorname{MSE}\left(\hat{\theta}_{c w}\right)=E\left(\hat{\theta}_{c w}-\theta\right)^{2}=E\left(\sum_{j=1}^{k} f_{c j} \hat{\theta}_{c j}-\theta\right)^{2}
$$

To obtain the minimum $Q$ subject to a constraint $\sum_{j=1}^{k} f_{c j}=1$, we form the auxiliary function $\phi$ to seek $f_{c j}$ that minimize

$$
\phi=E\left(\sum_{j=1}^{k} f_{c j} \hat{\theta}_{c j}-\theta\right)^{2}+\lambda\left(\sum_{j=1}^{k} f_{c j}-1\right)
$$

where $\lambda$ is a Lagrange multiplier. The weights $f_{c j}$ and $\lambda$ are derived by solving the following equations simultaneously: $\frac{\partial \phi}{\partial \lambda}=0, \frac{\partial \phi}{\partial f_{c j}}=0, j=1,2, \cdots, k$. The details are presented in Appendix. The result of the weighted estimate for the $j^{\text {th }}$ center yields

$$
\begin{aligned}
\hat{f}_{c j}= & \left(\frac{\hat{V}_{j}^{-1}\left(1+\hat{\tau}_{j} \hat{\theta}_{\text {pool }}\right)}{\hat{a}}\right) \\
& -\left(\frac{\hat{V}_{j}^{-1} \hat{\tau}_{j}}{\hat{a}+\sum_{m=1}^{k} \hat{\tau}_{m} \hat{E}_{m} \hat{V}_{m}^{-1}}\right)\left(\frac{\sum_{m=1}^{k} \hat{V}_{m}^{-1} \hat{E}_{m}\left(1+\hat{\tau}_{m} \hat{\theta}_{p o o l}\right)}{\hat{a}}\right)
\end{aligned}
$$

where $\hat{E}_{j}=\frac{n_{1 j} \hat{p}_{c 1 j}+c_{1}}{n_{1 j}+2 c_{1}}-\frac{n_{2 j} \hat{p}_{c 2 j}+c_{2}}{n_{2 j}+2 c_{2}}$

$\hat{V}_{j}=\frac{n_{1 j} \hat{p}_{c 1 j}\left(1-\hat{p}_{c 1 j}\right)}{\left(n_{1 j}+2 c_{1}\right)^{2}}+\frac{n_{2 j} \hat{p}_{c 2 j}\left(1-\hat{p}_{c 2 j}\right)}{\left(n_{2 j}+2 c_{2}\right)^{2}}, \hat{\tau}_{j}=\hat{a} \hat{E}_{j}-\hat{b}$

$\hat{a}=\sum_{j=1}^{k} \frac{1}{\hat{V}_{j}}=\sum_{j=1}^{k} \hat{V}_{j}^{-1}, \quad \hat{b}=\sum_{j=1}^{k} \frac{\hat{E}_{j}}{\hat{V}_{j}}, \quad \hat{\theta}_{\text {pool }}=\hat{p}_{1}-\hat{p}_{2}$

$\hat{p}_{1}=\frac{\sum_{j=1}^{k} n_{1 j} \hat{p}_{1 j}}{\sum_{j=1}^{k} n_{1 j}}=\frac{\sum_{j=1}^{k} X_{1 j}}{\sum_{j=1}^{k} n_{1 j}}$,

$\hat{p}_{2}=\frac{\sum_{j=1}^{k} n_{2 j} \hat{p}_{2 j}}{\sum_{j=1}^{k} n_{2 j}}=\frac{\sum_{j=1}^{k} X_{2 j}}{\sum_{j=1}^{k} n_{2 j}}$

In the particular case of $c_{1}=c_{2}=0$, our estimator $\hat{\theta}_{c w}=\sum_{j=1}^{k} f_{c j} \hat{\theta}_{c j}$ has a shrinkage estimator to be the popular inverse-variance weighted estimator. Under a common risk difference $\theta$ over all centers, the variance of $\hat{\theta}_{c w}$ in the case of non-random weights $f_{c j}$ are obtained by

$$
\begin{aligned}
V\left(\hat{\theta}_{c w}\right) & =\sum_{j=1}^{k} f_{c j}^{2} V\left(\hat{\theta}_{c j}\right) \\
& =\sum_{j=1}^{k} f_{c j}^{2}\left(\frac{n_{1 j} p_{1 j}\left(1-p_{1 j}\right)}{\left(n_{1 j}+2 c_{1}\right)^{2}}+\frac{n_{2 j} p_{2 j}\left(1-p_{2 j}\right)}{\left(n_{2 j}+2 c_{2}\right)^{2}}\right)
\end{aligned}
$$

Suppose that a normal approximation is reliable, the asymptotic distribution is

$$
\frac{\hat{\theta}_{c w}-\theta}{\sqrt{\hat{V}\left(\hat{\theta}_{c w}\right)}}=\frac{\sum_{j=1}^{k} \hat{f}_{c j} \hat{\theta}_{c j}-\theta}{\sqrt{\sum_{j=1}^{k} \hat{f}_{c j}^{2} \hat{V}\left(\hat{\theta}_{c j}\right)}} \rightarrow N(0,1)
$$


for testing $H_{0}: \theta=\theta_{0}$ we have the normal approximate test

$$
Z_{c w}=\frac{\sum_{j=1}^{k} \hat{f}_{c j} \hat{\theta}_{c j}-\theta_{0}}{\sqrt{\sum_{j=1}^{k} \hat{f}_{c j}^{2} \hat{V}\left(\hat{\theta}_{c j} \mid H_{0}\right)}}
$$

We will reject $H_{0}$ at $\alpha$ level for two-sided test if $\left|Z_{c w}\right|>Z_{\alpha / 2}$ where $Z_{\alpha / 2}$ is the upper $100\left(\alpha / 2^{\text {th }}\right)$ percentile of the standard normal distribution. Alternatively, $H_{0}$ is rejected when the p-value $(p)$ is less than or equal to $\alpha \quad(p \leq \alpha)$ where $\left.p=2 \quad 1-\Phi\left(\left|Z_{c w}\right|\right)\right]$ and $\Phi(Z)$ is the standard cumulative normal distribution function.

\section{Other Well-Known Weights}

\subsection{Cochran-Mantel-Haenszel (CMH) Weights}

Cochran $[10,11]$ proposed a weighted estimator of center-specific sample sizes for a common risk difference based on the unconditional binomial likelihood as

$$
\hat{\theta}_{C M H}=\frac{\sum_{j=1}^{k} w_{j} \hat{\theta}_{j}}{\sum_{j=1}^{k} w_{j}}
$$

where $w_{j}=\left(\frac{1}{n_{1 j}}+\frac{1}{n_{2 j}}\right)^{-1}=n_{1 j} n_{2 j} /\left(n_{1 j}+n_{2 j}\right)$ and

$\hat{\theta}_{j}=\hat{p}_{1 j}-\hat{p}_{2 j}=\frac{X_{1 j}}{n_{1 j}}-\frac{X_{2 j}}{n_{2 j}}$. Cochran's weight $w_{j}$ is

widely used as a standard non-random weight derived by the harmonic means of the center-specific sample sizes. Note that $f_{j}=w_{j} / \sum_{j=1}^{k} w_{j}$ is also Cochran's weight subject to the condition that $\sum_{j=1}^{k} f_{j}=1$. A straightforward derivation illustrates that $\hat{\theta}_{C M H}$ is an unbiased estimate of $\theta$ and the variance of $\hat{\theta}_{C M H}$ is readily available as

$$
V\left(\hat{\theta}_{C M H}\right)=\frac{\sum_{j=1}^{k} w_{j}^{2} V\left(\hat{\theta}_{j}\right)}{\left(\sum_{j=1}^{k} w_{j}\right)^{2}}
$$

where $V\left(\hat{\theta}_{j}\right)=p_{1 j}\left(1-p_{1 j}\right) / n_{1 j}+p_{2 j}\left(1-p_{2 j}\right) / n_{2 j}$. Assuming that a normal approximation is reliable, the Cochran's Z-statistic for testing $H_{0}: \theta=\theta_{0}$ is provided as

$$
Z_{C M H}=\frac{\left(\sum_{j=1}^{k} w_{j} \hat{\theta}_{j} / \sum_{j=1}^{k} w_{j}\right)-\theta_{0}}{\sqrt{\left(\sum_{j=1}^{k} w_{j}^{2} \hat{V}\left(\hat{\theta}_{j} \mid H_{0}\right)\right) /\left(\sum_{j=1}^{k} w_{j}\right)^{2}}}
$$

where $\hat{V}\left(\hat{\theta}_{j} \mid H_{0}\right)=\hat{p}_{1 j}\left(1-\hat{p}_{1 j}\right) / n_{1 j}+\hat{p}_{2 j}\left(1-\hat{p}_{2 j}\right) / n_{2 j}$. The rejection rule of $H_{0}$ follows the same as the previous standard normal test.

Alternatively, Mantel and Haenszel [12] suggested the test based on the conditional hypergeometric likelihood for a common odds ratio among the set of $k$ tables un- der the null hypothesis of $H_{0}: O R=1 \quad(\theta=0)$. With the null criterion, Mantel-Haenszel's weight stated by Sanchez-Meca and Marin-Martine [13] was equivalent to $w_{j}=n_{1 j} n_{2 j} /\left(n_{1 j}+n_{2 j}-1\right)$. Since the minor difference between the conditional Mantel-Haenszel weight and the unconditional Cochran weight is in the denominators, thus the two are often referred to interchangeably as the Cochran-Mantel-Haenszel weight. In this study, we use $w_{j}=n_{1 j} n_{2 j} /\left(n_{1 j}+n_{2 j}\right)$.

\subsection{Inverse Variance (INV) or Weighted Least Square (WLS) Weights}

Fleiss [14] and Lipsitz et al. [15] showed that the inverse-variance weighted (INV) estimator or the weightedleast-square (WLS) estimator for $\theta$ was in the summary estimator of the weighted mean (linear, unbiased estimator) of the form

$$
\hat{\theta}_{I N V}=\sum_{j=1}^{k} w_{j} \hat{\theta}_{j} / \sum_{j=1}^{k} w_{j}
$$

where $\hat{\theta}_{j}=\hat{p}_{1 j}-\hat{p}_{2 j}=X_{1 j} / n_{1 j}-X_{2 j} / n_{2 j}$ and $w_{j}$ defined by the reciprocal of the variance as

$$
w_{j}=\frac{1}{V\left(\hat{\theta}_{j}\right)}=\left(\frac{p_{1 j}\left(1-p_{1 j}\right)}{n_{1 j}}+\frac{p_{2 j}\left(1-p_{2 j}\right)}{n_{2 j}}\right)^{-1}
$$

The non-random and non-negative weights $w_{j}$ yield the minimum variance of the summary estimator $\hat{\theta}_{I N V}$ for estimating $\theta$. The variance of $\hat{\theta}_{I N V}$ is just given by

$$
V\left(\hat{\theta}_{I N V}\right)=\frac{\sum_{j=1}^{k} w_{j}^{2} V\left(\hat{\theta}_{j}\right)}{\left(\sum_{j=1}^{k} w_{j}\right)^{2}}=\frac{\sum_{j=1}^{k} w_{j}^{2}\left[1 / w_{j}\right]}{\left(\sum_{j=1}^{k} w_{j}\right)^{2}}=\frac{1}{\sum_{j=1}^{k} w_{j}}
$$

However, the weights $w_{j}$ cannot be used in practice since $p_{1 j}$ and $p_{2 j}$ are unknown. Therefore, it has become common practice to replace them by their sample estimators. It yields

$$
\hat{w}_{j}^{-1}=\frac{\hat{p}_{1 j}\left(1-\hat{p}_{1 j}\right)}{n_{1 j}}+\frac{\hat{p}_{2 j}\left(1-\hat{p}_{2 j}\right)}{n_{2 j}}
$$

This weight was suggested in several textbooks of epidemiology such as Kleinbaum et al. [16] or in textbooks of meta-analysis such as Petitt [17]. We assume that a normal approximation is reliable; the inverse-variance weighted test statistic for testing $H_{0}: \theta=\theta_{0}$ is

$$
Z_{I N V}=\frac{\left(\sum_{j=1}^{k} \hat{w}_{j} \hat{\theta}_{j} / \sum_{j=1}^{k} \hat{w}_{j}\right)-\theta_{0}}{\sqrt{\left(1 / \sum_{j=1}^{k} \hat{w}_{j}\right)}}
$$

where $\hat{w}_{j}^{-1}=\hat{V}\left(\hat{\theta}_{j} \mid H_{0}\right)$. Also, the rule of $H_{0}$ rejection follows the same as the above standard normal test. 


\section{Monte Carlo Simulation}

We perform simulations for estimating a common risk difference $\theta$ and testing the null hypothesis $H_{0}: \theta=\theta_{0}$ in the similar plans as follows:

Parameters Setting: Let the common risk difference $\theta$ be some constants varying from 0 to 0.6 , with incremental steps of 0.1. Baseline proportion risks $p_{2 j}$ $\left\{p_{21}, p_{22}, \cdots, p_{2 k}\right\}$ in the control arm for the $j^{\text {th }}$ center $(j=1,2, \cdots, k)$ are generated from a uniform distribution over $[0,0.95-\theta]$. The correspondent proportion risks $p_{1 j}$ for the treatment arm in the $j^{\text {th }}$ center are obtained as $p_{1 j}=p_{2 j}+\theta$. For example, if $\theta=0.2$, then $p_{2 j} \sim U(0,0.75)$ and $p_{1 j}=p_{2 j}+\theta \sim U(0.2,0.95)$. The sample sizes $n_{1 j}$ and $n_{2 j}$ are varied as $4,8,16,32$, 100. The number of centers $k$ takes values $1,2,4,8,16$, 32.

Statistics: Binomial random variables $X_{1 j}$ and $X_{2 j}$ in treatment and control arms are generated with parameters $\left(n_{1 j}, p_{1 j}\right)$ and $\left(n_{2 j}, p_{2 j}\right)$ for each center $j$.

Estimation: All summary estimates of $\theta$ are computed in a variety of different weights. The procedure is replicated 5000 times. From these replicates, bias, variance, and MSE (mean square error) are computed in the conventional way.

Type I Error: From the above parameter setting, we assign $\theta=\theta_{0}$ under a null $H_{0}: \theta=\theta_{0}$, so all tests are computed. The replication is treated 5000 times. From these replicates, the number of the null hypothesis rejecttions is counted for the empirical type I error $\hat{\alpha}$.

$$
\hat{\alpha}=\frac{\text { Number of rejections of } H_{0} \text { when } H_{0} \text { is true }}{\text { Number of replications (5000 times) }}
$$

The evaluation for two-sided tests in terms of the type I probability is based on Cochran limits [18] as follow.

At $\alpha=0.01$, the $\hat{\alpha}$ value is between $[0.005,0.015]$.

At $\alpha=0.05$, the $\hat{\alpha}$ value is between $[0.04,0.06]$.

At $\alpha=0.10$, the $\hat{\alpha}$ value is between $[0.08,0.12]$.

If the empirical type I error $\hat{\alpha}$ lies within those of Cochran limits, then the statistical test can control type I error.

Power of Tests: Before evaluating tests with their powers, all comparative tests should be calibrated to have the same type I error rate under $H_{0}$; then any test whose power hits the maximum under $H_{1}$ would be the best test. To achieve the alternative hypothesis, we assume the random effect model for $\theta_{j}$ as

$$
\theta_{j}=0.1+U_{m}=0.1+m(2 U-1)
$$

where $U_{m}$ as an effect of between centers is assigned to be uniform $[-m, m]$ for a given $m \in[0,0.1]$, or equivalently, $U$ is an uniform variable over $[0,1]$. That is, $E\left(\theta_{j}\right)=0.1$ and $\operatorname{Var}\left(\theta_{j}\right)=(2 m)^{2} / 12$. Also, we have $p_{1 j}=\theta_{j}+p_{2 j}$ where $p_{2 j}$ be uniform distri- bution over $[0.1,0.8]$. Binomial random variables $X_{1 j}$ and $X_{2 j}$ are drawn with parameters $\left(n_{1 j}, p_{1 j}\right)$, and $\left(n_{2 j}, p_{2 j}\right)$, respectively. All proposed test statistics are then computed. The procedure is replicated 5,000 times. From these replicates, the empirical power $1-\widehat{\beta}$ of test is counted.

$$
1-\widehat{\beta}=\frac{\text { Number of rejections of } H_{0} \text { when } H_{1} \text { is true }}{\text { Number of replications (5000 times) }}
$$

\section{Results}

Since it is difficult to present all enormous results from the simulation study, we just have illustrated some instances. Nevertheless, the main results are concluded perfectly.

\subsection{Results for Estimating Risk Differences}

Table 1 presents some results according to point estimation of a common risk difference $\theta$. However, we can draw conclusions in the following.

- The number of centers, $k$, can not change the order of the MSE of all weighted estimators, even though an increase in $k$ can decrease the variance and the MSE of all estimators, leading to the increasing efficiency. Also, increasing $n_{1 j}$ and $n_{2 j}$ can decrease the variance of all estimators while fixing $k$. The unbalanced cases of $n_{1 j}$ and $n_{2 j}$ for center $j$ have a rare effect on the order of the MSE of all estimates.

- For most popular situations used under $\theta=0$, $\theta=0.1, \theta=0.2$, and $\theta=0.3$, the proposed summary estimator $\hat{\theta}_{c w}$ adjusted by $c=c_{1}=c_{2}=1$ including adjusted by $c=2$ is the best choice with the smallest MSE. The estimator $\hat{\theta}_{c w}$ adjusted by $c=0.5$ and the inverse-variance (INV) weighted estimator $(c=0)$ are close together and are the second choice with smaller MSE. The Cochran-MantelHaenszel $(\mathrm{CMH})$ weight performs the worst in this simulation setting. This finding is very useful in general situations of most clinical trials and most causal relations between a disease and a suspected risk factor since the risk difference is often less than 0.25 [19].

- For $\theta=0.4$, the proposed estimator $\hat{\theta}_{c w}$ adjusted by $c=1$ performs best; for $\theta=0.5$, the proposed estimator $\hat{\theta}_{c w}$ adjusted by $c=0.5$ performs best; for $\theta=0.6$, the INV weighted estimator $(c=0)$ performs best.

\subsection{Results for Studying Type I Error}

Table 2 presents some results for controlling the empirical type I error. We can conclude the performance of several tests according to the empirical alpha under $H_{0}$ as follows. 
Table 1. Mean, variance, MSE for estimating $\theta$.

\begin{tabular}{|c|c|c|c|c|c|c|c|c|c|}
\hline$\theta$ & $k$ & $n_{1 j}$ & $n_{2 j}$ & Measure & $\mathrm{CMH}$ & $\begin{array}{c}\text { INV } \\
(c=0)\end{array}$ & $c=0.5$ & $c=1$ & $c=2$ \\
\hline \multirow[t]{3}{*}{0.0} & 1 & 2 & 2 & Mean: & -0.001700 & -0.000850 & -0.001130 & -0.000850 & -0.000570 \\
\hline & & & & Var: & 0.171245 & 0.042811 & 0.076109 & 0.042811 & 0.019027 \\
\hline & & & & MSE: & 0.171250 & 0.042813 & 0.076112 & 0.042813 & 0.019028 \\
\hline \multirow[t]{3}{*}{0.0} & 1 & 4 & 4 & Mean: & -0.000800 & 0.000400 & -0.000640 & -0.000530 & -0.000400 \\
\hline & & & & Var: & 0.088874 & 0.053058 & 0.056879 & 0.039499 & 0.022219 \\
\hline & & & & MSE: & 0.088875 & 0.053058 & 0.056880 & 0.039500 & 0.022219 \\
\hline \multirow[t]{3}{*}{0.0} & 1 & 8 & 8 & Mean: & 0.002625 & 0.001965 & 0.002333 & 0.002100 & 0.001750 \\
\hline & & & & Var: & 0.042575 & 0.035480 & 0.033641 & 0.027249 & 0.018923 \\
\hline & & & & MSE: & 0.042584 & 0.035483 & 0.033647 & 0.027254 & 0.018926 \\
\hline \multirow[t]{3}{*}{0.0} & 1 & 16 & 16 & Mean: & -0.000050 & 0.000328 & -0.000047 & -0.000044 & -0.000040 \\
\hline & & & & Var: & 0.021759 & 0.020761 & 0.019275 & 0.017193 & 0.013926 \\
\hline & & & & MSE: & 0.021759 & 0.020761 & 0.019275 & 0.017193 & 0.013926 \\
\hline \multirow[t]{3}{*}{0.0} & 1 & 32 & 32 & Mean: & -0.001900 & -0.001950 & -0.001840 & -0.001790 & -0.001690 \\
\hline & & & & Var: & 0.010805 & 0.010674 & 0.010160 & 0.009572 & 0.008538 \\
\hline & & & & MSE: & 0.010809 & 0.010678 & 0.010164 & 0.009575 & 0.008540 \\
\hline \multirow[t]{3}{*}{0.0} & 1 & 100 & 100 & Mean: & 0.000566 & 0.000572 & 0.000560 & 0.000555 & 0.000544 \\
\hline & & & & Var: & 0.003482 & 0.003478 & 0.003413 & 0.003346 & 0.003219 \\
\hline & & & & MSE: & 0.003482 & 0.003478 & 0.003413 & 0.003347 & 0.003219 \\
\hline \multirow[t]{3}{*}{0.1} & 16 & 2 & 2 & Mean: & 0.102200 & 0.051100 & 0.068133 & 0.051100 & 0.034067 \\
\hline & & & & Var: & 0.178755 & 0.044689 & 0.079446 & 0.044689 & 0.019861 \\
\hline & & & & MSE: & 0.178759 & 0.047080 & 0.080462 & 0.047080 & 0.024210 \\
\hline \multirow[t]{3}{*}{0.1} & 16 & 4 & 4 & Mean: & 0.101900 & 0.071067 & 0.081520 & 0.067933 & 0.050950 \\
\hline & & & & Var: & 0.093292 & 0.056358 & 0.059708 & 0.041462 & 0.023323 \\
\hline & & & & MSE: & 0.093295 & 0.057194 & 0.060047 & 0.042490 & 0.025729 \\
\hline \multirow[t]{3}{*}{0.1} & 16 & 4 & 8 & Mean: & 0.091175 & 0.073915 & 0.078964 & 0.069820 & 0.056883 \\
\hline & & & & Var: & 0.068527 & 0.048536 & 0.047903 & 0.036184 & 0.023445 \\
\hline & & & & MSE: & 0.068605 & 0.049217 & 0.048345 & 0.037095 & 0.025305 \\
\hline \multirow[t]{3}{*}{0.1} & 16 & 4 & 16 & Mean: & 0.096425 & 0.086770 & 0.087330 & 0.080322 & 0.069865 \\
\hline & & & & Var: & 0.057752 & 0.041273 & 0.040889 & 0.032469 & 0.024164 \\
\hline & & & & MSE: & 0.057764 & 0.041448 & 0.041048 & 0.032856 & 0.025072 \\
\hline \multirow[t]{3}{*}{0.1} & 16 & 4 & 32 & Mean: & 0.103087 & 0.094537 & 0.095306 & 0.089488 & 0.080958 \\
\hline & & & & Var: & 0.052651 & 0.037007 & 0.037127 & 0.030458 & 0.025400 \\
\hline & & & & MSE: & 0.052662 & 0.037037 & 0.037149 & 0.030568 & 0.025763 \\
\hline \multirow[t]{3}{*}{0.1} & 16 & 8 & 8 & Mean: & 0.105625 & 0.091604 & 0.093890 & 0.084500 & 0.070417 \\
\hline & & & & Var: & 0.047621 & 0.041375 & 0.037626 & 0.030478 & 0.021165 \\
\hline & & & & MSE: & 0.047653 & 0.041446 & 0.037664 & 0.030718 & 0.022040 \\
\hline \multirow[t]{3}{*}{0.1} & 16 & 8 & 16 & Mean: & 0.100700 & 0.094838 & 0.093524 & 0.087382 & 0.077367 \\
\hline & & & & Var: & 0.035620 & 0.031899 & 0.029404 & 0.024987 & 0.019128 \\
\hline & & & & MSE: & 0.035620 & 0.031926 & 0.029445 & 0.025147 & 0.019641 \\
\hline \multirow[t]{3}{*}{0.1} & 16 & 8 & 32 & Mean: & 0.097381 & 0.093334 & 0.092488 & 0.088258 & 0.081217 \\
\hline & & & & Var: & 0.028539 & 0.025407 & 0.023764 & 0.020808 & 0.017542 \\
\hline & & & & MSE: & 0.028546 & 0.025452 & 0.023820 & 0.020945 & 0.017895 \\
\hline \multirow[t]{3}{*}{0.1} & 16 & 16 & 16 & Mean: & 0.099100 & 0.094834 & 0.093271 & 0.088089 & 0.079280 \\
\hline & & & & Var: & 0.023792 & 0.023050 & 0.021075 & 0.018798 & 0.015227 \\
\hline & & & & MSE: & 0.023793 & 0.023077 & 0.021120 & 0.018941 & 0.015656 \\
\hline \multirow[t]{3}{*}{0.1} & 16 & 32 & 32 & Mean: & 0.100794 & 0.099611 & 0.097741 & 0.094866 & 0.089594 \\
\hline & & & & Var: & 0.011022 & 0.010951 & 0.010364 & 0.009764 & 0.008709 \\
\hline & & & & MSE: & 0.011023 & 0.010951 & 0.010369 & 0.009790 & 0.008817 \\
\hline \multirow[t]{3}{*}{0.1} & 16 & 100 & 100 & Mean: & 0.100052 & 0.099934 & 0.099061 & 0.098092 & 0.096204 \\
\hline & & & & Var: & 0.003728 & 0.003725 & 0.003654 & 0.003583 & 0.003446 \\
\hline & & & & MSE: & 0.003728 & 0.003725 & 0.003655 & 0.003587 & 0.003461 \\
\hline
\end{tabular}


Table 2. Empirical type I error for testing $H_{0}: \theta=\theta_{0}$ at $5 \%$ significance level.

\begin{tabular}{|c|c|c|c|c|c|c|c|c|}
\hline$\theta_{0}$ & $k$ & $n_{1 j}$ & $n_{2 j}$ & $\mathrm{CMH}$ & $\operatorname{INV}(c=0)$ & $c=0.5$ & $c=1$ & $c=2$ \\
\hline \multirow[t]{15}{*}{0.0} & 1 & 4 & 4 & 3.42 & 3.42 & 3.42 & 3.42 & 3.42 \\
\hline & & 4 & 8 & 2.08 & 2.08 & 6.84 & 4.76 & 4.76 \\
\hline & & 4 & 16 & 3.00 & 3.00 & 6.52 & 5.80 & 8.18 \\
\hline & & 4 & 32 & 2.76 & 2.76 & 6.66 & 6.18 & 10.50 \\
\hline & & 4 & 100 & 2.54 & 2.54 & 7.30 & 6.46 & 14.40 \\
\hline & & 8 & 8 & 3.28 & 3.28 & 6.76 & 4.16 & 4.16 \\
\hline & & 8 & 16 & 4.26 & 4.26 & 6.54 & 4.74 & 4.30 \\
\hline & & 8 & 32 & 4.34 & 4.34 & 5.58 & 4.22 & 5.10 \\
\hline & & 8 & 100 & 5.02 & 5.02 & 6.58 & 6.00 & 8.90 \\
\hline & & 16 & 16 & 4.74 & 4.74 & 4.48 & 4.48 & 3.38 \\
\hline & & 16 & 32 & 4.50 & 4.50 & 4.94 & 4.44 & 3.90 \\
\hline & & 16 & 100 & 5.02 & 5.02 & 5.30 & 4.58 & 5.10 \\
\hline & & 32 & 32 & 5.04 & 5.04 & 4.66 & 4.34 & 3.88 \\
\hline & & 32 & 100 & 5.22 & 5.22 & 5.16 & 4.46 & 4.34 \\
\hline & & 100 & 100 & 4.74 & 4.74 & 4.60 & 4.40 & 4.14 \\
\hline \multirow[t]{8}{*}{0.0} & 4 & 4 & 4 & 3.68 & 3.68 & 3.68 & 3.68 & 3.68 \\
\hline & & 8 & 8 & 3.40 & 3.40 & 7.14 & 4.56 & 4.56 \\
\hline & & 16 & 16 & 4.84 & 4.84 & 4.66 & 4.66 & 3.54 \\
\hline & & 16 & 32 & 4.52 & 4.52 & 5.00 & 4.52 & 4.10 \\
\hline & & 16 & 100 & 5.46 & 5.46 & 5.66 & 4.72 & 5.26 \\
\hline & & 32 & 32 & 4.74 & 4.74 & 4.42 & 4.18 & 3.92 \\
\hline & & 32 & 100 & 5.34 & 5.34 & 5.48 & 4.74 & 4.46 \\
\hline & & 100 & 100 & 5.04 & 5.04 & 4.98 & 4.86 & 4.64 \\
\hline \multirow[t]{8}{*}{0.1} & 4 & 4 & 4 & 1.26 & 1.26 & 8.28 & 8.28 & 6.22 \\
\hline & & 8 & 8 & 4.24 & 4.24 & 7.6 & 4.66 & 4.66 \\
\hline & & 16 & 16 & 5.18 & 5.18 & 5.76 & 5.04 & 4.06 \\
\hline & & 16 & 32 & 5.66 & 5.66 & 5.82 & 5.40 & 5.30 \\
\hline & & 16 & 100 & 58.6 & 5.86 & 62.0 & 4.84 & 4.88 \\
\hline & & 32 & 32 & 5.72 & 5.72 & 5.64 & 4.96 & 4.44 \\
\hline & & 32 & 100 & 5.88 & 5.88 & 5.44 & 5.20 & 4.82 \\
\hline & & 100 & 100 & 5.22 & 5.22 & 5.16 & 5.10 & 4.82 \\
\hline \multirow[t]{8}{*}{0.2} & 4 & 4 & 4 & 1.74 & 1.74 & 4.36 & 4.36 & 8.00 \\
\hline & & 8 & 8 & 4.66 & 4.66 & 8.58 & 5.38 & 5.38 \\
\hline & & 16 & 16 & 7.54 & 7.54 & 6.32 & 6.28 & 6.58 \\
\hline & & 16 & 32 & 7.26 & 7.26 & 6.22 & 5.56 & 5.60 \\
\hline & & 16 & 100 & 6.24 & 6.24 & 6.18 & 5.40 & 5.88 \\
\hline & & 32 & 32 & 5.46 & 5.46 & 5.40 & 5.46 & 5.08 \\
\hline & & 32 & 100 & 5.56 & 5.56 & 5.26 & 5.22 & 48.8 \\
\hline & & 100 & 100 & 5.34 & 5.34 & 5.16 & 5.10 & 5.22 \\
\hline \multirow[t]{8}{*}{0.4} & 4 & 4 & 4 & 3.00 & 3.00 & 12.06 & 7.44 & 18.04 \\
\hline & & 8 & 8 & 8.00 & 8.00 & 6.82 & 9.18 & 12.04 \\
\hline & & 16 & 16 & 5.78 & 5.78 & 5.92 & 5.16 & 7.04 \\
\hline & & 16 & 32 & 6.82 & 6.82 & 6.56 & 6.16 & 7.56 \\
\hline & & 16 & 100 & 6.38 & 6.38 & 6.18 & 5.80 & 7.06 \\
\hline & & 32 & 32 & 5.96 & 5.96 & 5.78 & 5.94 & 6.28 \\
\hline & & 32 & 100 & 5.92 & 5.92 & 5.80 & 6.04 & 6.72 \\
\hline & & 100 & 100 & 5.68 & 5.68 & 5.34 & 5.14 & 5.48 \\
\hline
\end{tabular}

Bold values denote that the statistical tests can control the type I error. 
- The increasing $k$ cannot change the order of the empirical type I error rates of all tests. Also, the unbalanced cases of $n_{1 j}$ and $n_{2 j}$ for center $j$ have a slight effect on the order of the empirical type I error rates of all tests.

- None of tests can control type I error rates when sample size of treatment or control arm is very small $\left(n_{1 j} \leq 4\right.$ or $\left.n_{2 j} \leq 4\right)$. There exists few tests that can control type I error when sample size is small $\left(n_{1 j}=8\right.$ or $\left.n_{2 j}=8\right)$.

- For $\theta=0$, almost all tests can control type I error rates when the sample size is moderate to large ( $n_{1 j} \geq 16$ or $n_{2 j} \geq 16$ ). This finding frequently occurs in practical use of $H_{0}: \theta=0$.

- For $\theta=0.2, \theta=0.4$, and $\theta=0.6$, almost all tests can control type I error rates when the sample size is large to very large $\left(n_{1 j} \geq 32\right.$ or $\left.n_{2 j} \geq 32\right)$.

\subsection{Results for Studying Power of Tests}

Table 3 shows some more details of the powers. Fortunately, almost all tests under $H_{0}: \theta=0$ can control type I error rates when the sample size is moderate to large ( $n_{1 j} \geq 16$ or $n_{2 j} \geq 16$ ). We ignore to consider the comparative tests when sample size is very small $\left(n_{1 j} \leq 4\right.$ or $n_{2 j} \leq 4$ ) since all of tests can not control type I error rates. The performance of several weighted tests according to the powers under $H_{1}: \theta_{j}=0.1+U_{m}$ can be concluded in the following:

- The empirical powers yield a similar pattern of results like the MSE. An increase in the number of centers, $k$, can increase the power but it can not change the order of power.

- Overall, the proposed weights adjusted by $c=1$ including $c=2$ perform best with the highest power in a multi-center study of size $k \geq 2$ when $n_{1 j} \geq 16$ or $n_{2 j} \geq 16$.

- The INV weight and the CMH weight are achieved with the highest powers in one center study when $n_{1 j} \geq 16$ or $n_{2 j} \geq 16$.

- When the sample size is large to very large $\left(n_{1 j} \geq 32\right.$ or $n_{2 j} \geq 32$ ), all weights perform well.

\section{Numerical Examples}

Two examples are presented to illustrate the implementation of the related methodology. Pocock [20] presented data from a randomized trial studying the effect of placebo and metoprolol on mortality after heart attack (AMI: Acute Myocardial Infarction) classified by three strata of age groups, namely, 40 - 64, 65 - 69, 70 - 74 years. Table 4 shows the data and weights corresponding to the $\mathrm{CMH}$, the INV, and the proposed strategies. The estimated summary differences based on the $\mathrm{CMH}$, the INV, and the proposed weights are $0.031,0.024,0.030$, re- spectively. Also, the estimated standard errors of those of overall differences are $0.014,0.013,0.014$, respectively. Since both of $Z_{C M H}=2.237$ and $Z_{c w}=2.197$ are greater than $Z_{\alpha / 2}=1.96$, the $\mathrm{CMH}$ and the proposed tests at $c=1$ reject the null hypothesis at 5\% level for two-sided test and lead to the conclusion of a significant difference between the placebo and metoprolol mortality rates whereas the INV test with $Z_{I N V}=1.823$ fails to reject the null hypothesis at 5\% level.

Turner et al. [21] presented data from clinical trials to study the effect of selective decontamination of the digestive tract on the risk of respiratory tract infection of patients in intensive care units. See data and weights in Table 5. The estimated overall differences and their estimated standard errors are 0.152 (0.012), 0.140 (0.011), $0.162(0.012)$ for the $\mathrm{CMH}$, the INV, and the proposed weights at $c=1$, respectively. All tests reject the null hypothesis with $Z_{C M H}=12.584, Z_{I N V}=12.215$, $Z_{c w}=13.719$ and lead to the conclusion of a significant difference between treatment effect of selective decontamination of the digestive tract on the risk of respiratory tract infection.

\section{Conclusions and Discussion}

In most general situations used by the risk difference lying on $[0,0.25]$, the results have confirmed that the minimum MSE weight of the proposed summary estimator $\hat{\theta}_{c w}$ adjusted by $c=c_{1}=c_{2}=1$ (including $\left.c=c_{1}=c_{2}=2\right)$ is the best choice with the smallest MSE under a constant of common risk difference $\theta$ over all $k$ centers. The number of centers, $k$, cannot change the order of the MSE of all weighted estimators, even though an increase in $k$ can decrease the variance and the MSE of all weighted estimators. Also, increasing $n_{1 j}$ and $n_{2 j}$ can decrease the variance of all estimators while fixing $k$. The unbalanced cases of $n_{1 j}$ and $n_{2 j}$ for center $j$ have a slight effect on the order of the MSE of all estimates. The minimum MSE weight is designed to yield more precise estimate relative to the $\mathrm{CMH}$ and INV weights. Another benefit of the proposed weight is easy to compute because of its closed-form formula. With the basis of smallest MSE and the easy-to-compute formula, we have been solidly suggested to use the proposed weight. In addition, the various choices for $c$ have been considered again. The use of $c=0.5$ as a conventional correction term [22] should be revised. The better value of $c$ in adding on the number of successes and the number of failures is suggested with at least for $c=1$ (including $c=2$ ). This result is supported by the ideas of Böhning and Viwatwongkasem [6], Agresti and Coull [1], and Agresti and Caffol [2] that recommended to use the appropriate values of $c$ greater than or equal to 1. 
Table 3. Empirical power (percent) at $\boldsymbol{m}=\mathbf{0 . 0 4}$ after controlling the estimated type I error at the nominal $5 \%$ level.

\begin{tabular}{|c|c|c|c|c|c|c|c|c|c|c|c|c|}
\hline \multirow[b]{2}{*}{$k$} & \multirow[b]{2}{*}{$n_{1 j}$} & \multirow[b]{2}{*}{$n_{2 j}$} & \multicolumn{5}{|c|}{$\mathrm{X}=$ Controllable Type I error rates } & \multicolumn{5}{|c|}{ Empirical power rates } \\
\hline & & & $\mathrm{CMH}$ & INV & $c=0.5$ & $c=1$ & $c=2$ & $\mathrm{CMH}$ & INV & $c=0.5$ & $c=1$ & $c=2$ \\
\hline \multirow[t]{10}{*}{1} & 8 & 8 & & & & $\mathrm{X}$ & $\mathrm{X}$ & & & & 6.8 & 6.8 \\
\hline & 8 & 16 & $\mathrm{X}$ & $\mathrm{X}$ & & $\mathrm{X}$ & $\mathrm{X}$ & 7.3 & 7.3 & & 8.3 & 7.4 \\
\hline & 8 & 32 & $\mathrm{X}$ & $\mathrm{X}$ & $\mathrm{X}$ & $\mathrm{X}$ & $\mathrm{X}$ & 9.5 & 9.5 & 11.5 & 9.6 & 9.9 \\
\hline & 8 & 100 & $\mathrm{X}$ & $\mathrm{X}$ & & $\mathrm{X}$ & & 11.3 & 11.3 & & 11.8 & \\
\hline & 16 & 16 & $\mathrm{X}$ & $\mathrm{X}$ & $\mathrm{X}$ & $\mathrm{X}$ & & 11.2 & 11.2 & 10.6 & 10.6 & \\
\hline & 16 & 32 & $\mathrm{X}$ & $\mathrm{X}$ & $\mathrm{X}$ & $\mathrm{X}$ & & 12.2 & 12.2 & 12.7 & 11.8 & \\
\hline & 16 & 100 & $X$ & $X$ & $X$ & $X$ & $X$ & 16.4 & 16.4 & 15.4 & 14.8 & 14.6 \\
\hline & 32 & 32 & $\mathrm{X}$ & $X$ & $X$ & $X$ & & 17.6 & 17.6 & 16.5 & 16.4 & \\
\hline & 32 & 100 & $X$ & $X$ & $X$ & $X$ & $X$ & 21.4 & 21.4 & 21.2 & 20.8 & 20.3 \\
\hline & 100 & 100 & $\mathrm{X}$ & $X$ & $X$ & $X$ & $\mathrm{X}$ & 36.8 & 36.8 & 36.8 & 36.5 & 36.1 \\
\hline \multirow[t]{10}{*}{4} & 8 & 8 & & & & $X$ & $X$ & & & & 26.9 & 29.7 \\
\hline & 8 & 16 & & & & $X$ & $\mathrm{X}$ & & & & 29.5 & 32.8 \\
\hline & 8 & 32 & $X$ & $X$ & $X$ & $X$ & $\mathrm{X}$ & 20.8 & 23.8 & 31.5 & 33.1 & 35.2 \\
\hline & 8 & 100 & $X$ & $X$ & & $\mathrm{X}$ & & 23.6 & 28.9 & & 36.8 & \\
\hline & 16 & 16 & $\mathrm{X}$ & $X$ & $X$ & $X$ & & 25.3 & 27.0 & 31.0 & 33.4 & \\
\hline & 16 & 32 & $X$ & $X$ & $X$ & $X$ & $X$ & 32.4 & 35.9 & 38.0 & 40.6 & 43.6 \\
\hline & 16 & 100 & $X$ & $X$ & $X$ & $X$ & $\mathrm{X}$ & 39.1 & 44.6 & 45.2 & 46.8 & 48.9 \\
\hline & 32 & 32 & $X$ & $X$ & $X$ & $X$ & & 44.2 & 46.1 & 47.8 & 49.5 & \\
\hline & 32 & 100 & $X$ & $X$ & $X$ & $X$ & $X$ & 58.1 & 60.6 & 61.4 & 62.8 & 64.6 \\
\hline & 100 & 100 & $X$ & $X$ & $X$ & $X$ & $X$ & 85.6 & 87.0 & 86.7 & 87.2 & 87.8 \\
\hline \multirow[t]{10}{*}{8} & 8 & 8 & & & & & & & & & & \\
\hline & 8 & 16 & & & $X$ & $X$ & & & & 44.0 & 48.8 & \\
\hline & 8 & 32 & $\mathrm{X}$ & $X$ & $X$ & $\mathrm{X}$ & $\mathrm{X}$ & 35.3 & 39.5 & 50.2 & 53.9 & 59.0 \\
\hline & 8 & 100 & $X$ & $X$ & & & & 39.9 & 46.0 & & & \\
\hline & 16 & 16 & $X$ & $X$ & $X$ & $X$ & & 43.1 & 45.9 & 52.7 & 56.9 & \\
\hline & 16 & 32 & $X$ & $X$ & $X$ & $X$ & $\mathrm{X}$ & 53.4 & 57.0 & 61.3 & 64.3 & 68.5 \\
\hline & 16 & 100 & $X$ & $X$ & $X$ & $X$ & $\mathrm{X}$ & 65.7 & 69.3 & 72.0 & 74.5 & 77.1 \\
\hline & 32 & 32 & $X$ & $X$ & $X$ & $X$ & $X$ & 71.1 & 72.9 & 74.8 & 76.9 & 80.4 \\
\hline & 32 & 100 & $X$ & $X$ & $X$ & $X$ & $X$ & 86.1 & 87.7 & 88.3 & 89.1 & 90.4 \\
\hline & 100 & 100 & $X$ & $X$ & $X$ & $X$ & $X$ & 98.8 & 98.9 & 99.0 & 99.1 & 99.1 \\
\hline \multirow[t]{10}{*}{16} & 8 & 8 & & & & $X$ & $X$ & & & & 68.3 & 77.5 \\
\hline & 8 & 16 & & & $X$ & & & & & 68.5 & & \\
\hline & 8 & 32 & $\mathrm{X}$ & $X$ & $X$ & $X$ & $\mathrm{X}$ & 60.9 & 64.6 & 74.2 & 77.1 & 82.1 \\
\hline & 8 & 100 & $X$ & $X$ & & $X$ & & 67.4 & 72.1 & & 82.0 & \\
\hline & 16 & 16 & $X$ & $X$ & $X$ & $X$ & & 71.0 & 73.8 & 79.0 & 82.2 & \\
\hline & 16 & 32 & $\mathrm{X}$ & $\mathrm{X}$ & $X$ & $X$ & $\mathrm{X}$ & 82.5 & 84.4 & 87.3 & 89.2 & 92.0 \\
\hline & 16 & 100 & $\mathrm{X}$ & $X$ & $X$ & $X$ & $\mathrm{X}$ & 90.3 & 90.8 & 93.1 & 93.8 & 94.8 \\
\hline & 32 & 32 & $X$ & $X$ & $X$ & $X$ & & 93.9 & 94.9 & 95.3 & 96.0 & \\
\hline & 32 & 100 & $\mathrm{X}$ & $X$ & $X$ & $\mathrm{X}$ & $\mathrm{X}$ & 99.0 & 99.1 & 99.1 & 99.2 & 99.3 \\
\hline & 100 & 100 & $\mathrm{X}$ & $\mathrm{X}$ & $\mathrm{X}$ & $\mathrm{X}$ & $\mathrm{X}$ & 100.0 & 100.0 & 100.0 & 100.0 & 100.0 \\
\hline \multirow[t]{10}{*}{32} & 8 & 8 & & & & & & & & & & \\
\hline & 8 & 16 & $\mathrm{X}$ & $X$ & & $\mathrm{X}$ & $\mathrm{X}$ & 81.8 & 83.2 & & 92.7 & 95.6 \\
\hline & 8 & 32 & $\mathrm{X}$ & $\mathrm{X}$ & $\mathrm{X}$ & $\mathrm{X}$ & $\mathrm{X}$ & 88.7 & 90.0 & 94.1 & 95.1 & 96.7 \\
\hline & 8 & 100 & $\mathrm{X}$ & $\mathrm{X}$ & & $\mathrm{X}$ & & 92.2 & 93.4 & & 96.4 & \\
\hline & 16 & 16 & $\mathrm{X}$ & $X$ & & & & 94.5 & 95.0 & & 97.5 & \\
\hline & 16 & 32 & $\mathrm{X}$ & $X$ & $X$ & $\mathrm{X}$ & & 98.1 & 98.5 & 99.0 & 99.1 & \\
\hline & 16 & 100 & $X$ & $X$ & $X$ & $\mathrm{X}$ & $\mathrm{X}$ & 99.7 & 99.5 & 99.8 & 99.9 & 99.9 \\
\hline & 32 & 32 & $X$ & $X$ & $X$ & $X$ & & 99.8 & 99.9 & 99.9 & 99.9 & \\
\hline & 32 & 100 & $\mathrm{X}$ & $\mathrm{X}$ & $\mathrm{X}$ & $\mathrm{X}$ & $\mathrm{X}$ & 100.0 & 100.0 & 100.0 & 100.0 & 100.0 \\
\hline & 100 & 100 & $X$ & $X$ & $X$ & $X$ & $X$ & 100.0 & 100.0 & 100.0 & 100.0 & 100.0 \\
\hline
\end{tabular}


Table 4. Mortality data over three strata of age groups following Pocock.

\begin{tabular}{cccccccccc}
\hline Age & \multicolumn{2}{c}{ Placebo } & \multicolumn{2}{c}{ Metoprolol } & \multicolumn{3}{c}{ Weights } \\
\hline$j$ & $x_{1 j}$ & $n_{1 j}$ & $x_{2 j}$ & $n_{2 j}$ & $\hat{\theta}_{j}$ & CMH & INV & $c=1$ & 0.69 \\
\hline $40-64$ & 26 & 453 & 21 & 464 & 0.012 & 0.66 & 0.79 & 0.16 & 0.25 \\
$65-69$ & 25 & 174 & 11 & 165 & 0.077 & 0.24 & 0.10 & 0.05 & 0.06 \\
$70-74$ & 11 & 70 & 8 & 69 & 0.041 & 0.10 & \\
\hline
\end{tabular}

Table 5. Respiratory tract infections following Turner et al.

\begin{tabular}{|c|c|c|c|c|c|c|c|c|}
\hline \multirow{2}{*}{$\begin{array}{c}\text { Trial } \\
j\end{array}$} & \multicolumn{2}{|c|}{ Treatment I } & \multicolumn{2}{|c|}{ Treatment II } & \multirow[b]{2}{*}{$\hat{\theta}_{j}$} & \multicolumn{3}{|c|}{ Weights } \\
\hline & $x_{1 j}$ & $n_{1 j}$ & $x_{2 j}$ & $n_{2 j}$ & & $\mathrm{CMH}$ & INV & $c=1$ \\
\hline 1 & 25 & 54 & 7 & 47 & 0.314 & 0.03 & 0.02 & 0.02 \\
\hline 2 & 24 & 41 & 4 & 38 & 0.480 & 0.02 & 0.02 & 0.03 \\
\hline 3 & 37 & 95 & 20 & 96 & 0.181 & 0.05 & 0.03 & 0.04 \\
\hline 4 & 11 & 17 & 1 & 14 & 0.576 & 0.01 & 0.01 & 0.01 \\
\hline 5 & 26 & 49 & 10 & 48 & 0.322 & 0.03 & 0.02 & 0.02 \\
\hline 6 & 13 & 84 & 2 & 101 & 0.135 & 0.05 & 0.07 & 0.07 \\
\hline 7 & 38 & 170 & 12 & 161 & 0.149 & 0.09 & 0.09 & 0.09 \\
\hline 8 & 29 & 60 & 1 & 28 & 0.448 & 0.02 & 0.02 & 0.03 \\
\hline 9 & 9 & 20 & 1 & 19 & 0.397 & 0.01 & 0.01 & 0.01 \\
\hline 10 & 44 & 47 & 22 & 49 & 0.487 & 0.03 & 0.02 & 0.03 \\
\hline 11 & 30 & 160 & 25 & 162 & 0.033 & 0.08 & 0.07 & 0.06 \\
\hline 12 & 40 & 185 & 31 & 200 & 0.061 & 0.10 & 0.08 & 0.07 \\
\hline 13 & 10 & 41 & 9 & 39 & 0.013 & 0.02 & 0.01 & 0.01 \\
\hline 14 & 40 & 185 & 22 & 193 & 0.102 & 0.10 & 0.09 & 0.09 \\
\hline 15 & 4 & 46 & 0 & 45 & 0.087 & 0.02 & 0.06 & 0.04 \\
\hline 16 & 60 & 140 & 31 & 131 & 0.192 & 0.07 & 0.04 & 0.05 \\
\hline 17 & 12 & 75 & 4 & 75 & 0.107 & 0.04 & 0.05 & 0.05 \\
\hline 18 & 42 & 225 & 31 & 220 & 0.046 & 0.12 & 0.11 & 0.09 \\
\hline 19 & 26 & 57 & 7 & 55 & 0.329 & 0.03 & 0.02 & 0.03 \\
\hline 20 & 17 & 92 & 3 & 91 & 0.152 & 0.05 & 0.07 & 0.07 \\
\hline 21 & 23 & 23 & 14 & 25 & 0.440 & 0.01 & 0.01 & 0.02 \\
\hline 22 & 6 & 68 & 3 & 65 & 0.042 & 0.03 & 0.07 & 0.05 \\
\hline
\end{tabular}

In terms of type I error estimates, when sample size is very small $\left(n_{1 j} \leq 4\right.$ or $\left.n_{2 j} \leq 4\right)$, none of tests can control type I error rates. In addition, there exists few tests that can control type I error rates when sample size is small ( $n_{1 j}=8$ or $n_{2 j}=8$ ). This result is consonant with the comments of Lui [23] that none of conventional tests/weights under sparse data is appropriate. This inappropriateness under sparse data can cope with the minimum MSE weights from this finding. The further work to seek some appropriate tests/weights in sparse data challenges for investigators to develop an innovation or to improve much more reasonable tests/weights. In general results, almost all tests can control type I error rates when sample size is moderate to large $\left(n_{1 j} \geq 16\right.$ or $n_{2 j} \geq 16$ ).

In terms of power, we ignore to evaluate the power when sample size is very small $\left(n_{1 j} \leq 4\right.$ or $\left.n_{2 j} \leq 4\right)$ because all tests can not control type I error rates. The results illustrate the same pattern like the MSE. The proposed weights adjusted by $c=1$ including $c=2$ perform best with the highest power in a multi-center study of size $k \geq 2$ when $n_{1 j} \geq 16$ or $n_{2 j} \geq 16$. The INV weight and the CMH weight are achieved with the highest powers in one center study when $n_{1 j} \geq 16$ or $n_{2 j} \geq 16$. When sample size is large to very large ( $n_{1 j} \geq 32$ or $n_{2 j} \geq 32$ ), all tests perform well. We 
strongly recommend to use the minimum MSE weight as an appropriate choice because of its highest power.

\section{Acknowledgements}

We would like to thank the editors and the referees for comments which greatly improved this paper. This study was partially supported for publication by the China Medical Board (CMB), Faculty of Public Health, Mahidol University, Bangkok, Thailand.

\section{REFERENCES}

[1] A. Agresti and B. A. Coull, "Approximate Is Better than Exact for Interval Estimation of Binomial Proportions," American Statistical Association, Vol. 52, 1998, pp. 119126.

[2] A. Agresti and B. Caffo, "Simple and Effective Confidence Intervals for Proportions and Differences of Proportions Result from Adding Two Successes and Two Failures,” The American Statistician, Vol. 54, No. 4, 2000, pp. 280-288. doi:10.2307/2685779

[3] B. K. Ghosh, "A Comparison of Some Approximate Confidence Interval for the Binomial Parameter," Journal of the American Statistical Association, Vol. 74, No. 368, 1979, pp. 894-900. doi:10.2307/2286420

[4] R. G. Newcombe, "Two-Sided Confidence Intervals for the Single Proportion: Comparison of Seven Methods," Statistics in Medicine, Vol. 17, No. 8, 1998, pp. 857-872. doi:10.1002/(SICI)1097-0258(19980430)17:8<857::AIDSIM777>3.0.CO;2-E

[5] R. G. Newcombe, "Interval Estimation for the Difference between Independent Proportions: Comparison of Eleven Methods," Statistics in Medicine, Vol. 17, No. 8, 1998, pp. 873-890. doi:10.1002/(SICI)1097-0258(19980430)17:8<873::AIDSIM779>3.0.CO;2-I

[6] D. Böhning and C. Viwatwongkasem, "Revisiting Proportion Estimators," Statistical Methods in Medical Research, Vol. 14, No. 2, 2005, pp. 147-169. doi:10.1191/0962280205sm393oa

[7] G. Casella and R. L. Berger, "Statistical Inference,” Duxbury Press, Belmont, 1990.

[8] A. E. Taylor and W. R. Mann, "Advanced Calculus,” John Wiley \& Sons, New York, 1972.

[9] K. J. Lui and K. C. Chang, “Testing Homogeneity of Risk Difference in Stratified Randomized Trials with Noncompliance," Computational Statistics and Data Analysis, Vol. 53, No. 1, 2008, pp. 209-221. doi:10.1016/j.csda.2008.07.016

[10] W. G. Cochran, "The Combination of Estimates from Different Experiments,” Biometrics, Vol. 10, No. 1, 1954, pp. 101-129. doi:10.2307/3001666

[11] W. G. Cochran, "Some Methods for Strengthening the
Common Chi-Square Test,” Biometrics, Vol. 10, No. 4, 1954, pp. 417-451. doi:10.2307/3001616

[12] N. Mantel and W. Haenszel, "Statistical Aspects of the Analysis of Data from Retrospective Studies of Disease," Journal of the National Cancer Institute, Vol. 22, 1959, pp. 719-748.

[13] J. Sanchez-Meca and F. Marin-Martinez, "Testing the Significance of a Common Risk Difference in MetaAnalysis," Computational Statistics \& Data Analysis, Vol. 33, No. 3, 2000, pp. 299-313. doi:10.1016/S0167-9473(99)00055-9

[14] J. L. Fleiss, "Statistical Methods for Rates and Proportions,” John Wiley \& Sons Inc., New York, 1981.

[15] S. R. Lipsitz, K. B. G. Dear, N. M. Laird and G. Molenberghs, "Tests for Homogeneity of the Risk Difference When Data Are Sparse,” Biometrics, Vol. 54, No. 1, 1998, pp. 148-160. doi:10.2307/2534003

[16] D. G. Kleinbaum, L. L. Kupper and H. Morgenstern, "Epidemiologic Research: Principles and Quantitative Methods,” Lifetime Learning Publications, Belmont, 1982.

[17] D. B. Petitti, "Meta-Analysis, Decision Analysis and Cost-Effectiveness Analysis: Methods for Quantitative Synthesis in Medicine,” Oxford University Press, Oxford, 1994.

[18] W. G. Cochran, "The Chi-Square Test of Goodness of Fit,” Annals of Mathematical Statistics, Vol. 23, No. 3, 1952, pp. 315-345. doi:10.1214/aoms/1177729380

[19] K. J. Lui and C. Kelly, "Tests for Homogeneity of the Risk Ratio in a Series of $2 \times 2$ Tables," Statistics in Medicine, Vol. 19, No. 21, 2000, pp. 2919-2932. doi:10.1002/1097-0258(20001115)19:21<2919::AID-SI M561>3.0.CO;2-D

[20] S. J. Pocock, “Clinical Trials: A Practical Approach,” Wiley Publication, New York, 1997.

[21] R. Tuner, R. Omar, M. Yang, H. Goldstein and S. Thompson, "A Multilevel Model Framework for MetaAnalysis of Clinical Trials with Binary Outcome,” Statistics in Medicine, Vol. 19, No. 24, 2000, pp. 3417-3432. doi:10.1002/1097-0258(20001230)19:24<3417::AID-SI M614>3.0.CO;2-L

[22] F. Yates, "Contingency Tables Involving Small Numbers and the Chi-Squared Test," Journal of the Royal Statistical Society (Supplement), Vol. 1, 1934, pp. 217-235.

[23] K. J. Lui, “A Simple Test of the Homogeneity of Risk Difference in Sparse Data: An Application to a Multicenter Study,” Biometrical Journal, Vol. 47, No. 5, 2008, pp. 654-661. doi:10.1002/bimj.200410150

[24] C. A. Rencher, "Linear Models in Statistics,” Wiley Series in Probability and Mathematical Statistics, New York, 2000.

[25] A. Sen and M. Srivastava, "Regression Analysis: Theory, Methods, and Applications,” Springer-Verlag, New York, 1990. 


\section{Appendix}

Under a true common risk difference $\theta$ over all $k$ centers $(j=1,2, \cdots, k)$, the mean square error of $\hat{\theta}_{c w}=\sum_{j=1}^{k} f_{c j} \hat{\theta}_{c j}$ is given by

$$
\operatorname{MSE}\left(\hat{\theta}_{c w}\right)=E\left(\hat{\theta}_{c w}-\theta\right)^{2}=E\left(\sum_{j=1}^{k} f_{c j} \hat{\theta}_{c j}-\theta\right)^{2}
$$

To obtain the optimal weights $\left\{f_{c j}\right\}$ subject to a constraint that $\sum_{j=1}^{k} f_{c j}-1=0$, we form the auxiliary function $\phi$ by following Lagrange's method to seek $\left\{f_{c j}\right\}$ that minimize

$$
\begin{gathered}
\phi=E\left(\sum_{j=1}^{k} f_{c j} \hat{\theta}_{c j}-\theta\right)^{2}+\lambda\left(\sum_{j=1}^{k} f_{c j}-1\right) \\
\phi=E\left(\sum_{j=1}^{k} f_{c j} \hat{\theta}_{c j}\right)^{2}-2 \theta E\left(\sum_{j=1}^{k} f_{c j} \hat{\theta}_{c j}\right)+\theta^{2}+\lambda\left(\sum_{j=1}^{k} f_{c j}-1\right) \\
\phi=V\left(\sum_{j=1}^{k} f_{c j} \hat{\theta}_{c j}\right)+\left(E\left(\sum_{j=1}^{k} f_{c j} \hat{\theta}_{c j}\right)\right)^{2} \\
-2 \theta \sum_{j=1}^{k} f_{c j} E\left(\hat{\theta}_{c j}\right)+\theta^{2}+\lambda\left(\sum_{j=1}^{k} f_{c j}-1\right) \\
\phi=\sum_{j=1}^{k} f_{c j}^{2} V\left(\hat{\theta}_{c j}\right)+\left(\sum_{j=1}^{k} f_{c j} E\left(\hat{\theta}_{c j}\right)-\theta\right)^{2}+\lambda\left(\sum_{j=1}^{k} f_{c j}-1\right)
\end{gathered}
$$

Let $V_{j}=V\left(\hat{\theta}_{c j}\right)$ and $E_{j}=E\left(\hat{\theta}_{c j}\right)$. The partial derivatives with respect to $\lambda$ and $f_{c j}$ yield

$$
\frac{\partial \phi}{\partial \lambda}=\sum_{j=1}^{k} f_{c j}-1, \frac{\partial \phi}{\partial f_{c j}}=2 f_{c j} V_{j}+2\left(\sum_{j=1}^{k} f_{c j} E_{j}-\theta\right) E_{j}+\lambda
$$

Setting $\frac{\partial \phi}{\partial \lambda}=0$ and $\frac{\partial \phi}{\partial f_{c j}}=0$, it yields

$$
\begin{aligned}
& \sum_{j=1}^{k} f_{c j}=1 \\
& j=1 ; \quad\left(a V_{1}+E_{1} \tau_{1}\right) f_{c 1}+f_{c 2} E_{2} \tau_{1}+f_{c 3} E_{3} \tau_{1}+\cdots+f_{c k} E_{k} \tau_{1}=1+\tau_{1} \theta \\
& j=2 ; \quad f_{c 1} E_{1} \tau_{2}+\left(a V_{2}+E_{2} \tau_{2}\right) f_{c 2}+f_{c 3} E_{3} \tau_{2}+\cdots+f_{c k} E_{k} \tau_{2}=1+\tau_{2} \theta \\
& j=3 ; f_{c 1} E_{1} \tau_{3}+f_{c 2} E_{2} \tau_{3}+\left(a V_{3}+E_{3} \tau_{3}\right) f_{c 3}+\ldots+f_{c k} E_{k} \tau_{3}=1+\tau_{3} \theta \\
& j=k ; \quad f_{c 1} E_{1} \tau_{k}+f_{c 2} E_{2} \tau_{k}+f_{c 3} E_{3} \tau_{k}+\ldots+\left(a V_{k}+E_{k} \tau_{k}\right) f_{c k}=1+\tau_{k} \theta
\end{aligned}
$$
be written as

Hence,
Let $\tau_{j}=a E_{j}-b$

$$
\begin{gathered}
a V_{j} f_{c j}+\left(\sum_{j=1}^{k} f_{c j} E_{j}\right) \tau_{j}=1+\tau_{j} \theta \\
a V_{j} f_{c j}+\left(f_{c 1} E_{1}+f_{c 2} E_{2}+\cdots+f_{c k} E_{k}\right) \tau_{j}=1+\tau_{j} \theta
\end{gathered}
$$

Substitute each of the subscript $j$ and rearrange

It can be written in the matrix form as $\mathbf{H f}=\mathbf{y}$, where

$$
f_{c j}=-\frac{E_{j}}{V_{j}}\left(\sum_{j=1}^{k} f_{c j} E_{j}-\theta\right)-\frac{\lambda}{2 V_{j}}
$$

Solving for $\lambda$ by taking summation on $f_{c j}$, it yields

$$
\sum_{j=1}^{k} f_{c j}=1=-\left(\sum_{j=1}^{k} \frac{E_{j}}{V_{j}}\right)\left(\sum_{j=1}^{k} f_{c j} E_{j}-\theta\right)-\frac{\lambda}{2}\left(\sum_{j=1}^{k} \frac{1}{V_{j}}\right)
$$

Let $a=\sum_{j=1}^{k} \frac{1}{V_{j}}=\sum_{j=1}^{k} V_{j}^{-1}, \quad b=\sum_{j=1}^{k} \frac{E_{j}}{V_{j}}$, then $\lambda / 2$ can

$$
\frac{\lambda}{2}=-\frac{1}{a}-\frac{b\left(\sum_{j=1}^{k} f_{c j} E_{j}-\theta\right)}{a}
$$$$
\mathbf{H}=\left[\begin{array}{ccccc}
a V_{1}+E_{1} \tau_{1} & E_{2} \tau_{1} & E_{3} \tau_{1} & \cdots & E_{k} \tau_{1} \\
E_{1} \tau_{2} & a V_{2}+E_{2} \tau_{2} & E_{3} \tau_{2} & \cdots & E_{k} \tau_{2} \\
E_{1} \tau_{3} & E_{2} \tau_{3} & a V_{3}+E_{3} \tau_{3} & \cdots & E_{k} \tau_{3} \\
\vdots & \vdots & \vdots & \ddots & \vdots \\
E_{1} \tau_{k} & E_{2} \tau_{k} & E_{3} \tau_{k} & \cdots & a V_{k}+E_{k} \tau_{k}
\end{array}\right]
$$ 


$$
\begin{gathered}
\mathbf{f}^{\prime}=\left[\begin{array}{lllll}
f_{c 1} & f_{c 2} & f_{c 3} & \cdots & f_{c k}
\end{array}\right], \\
\mathbf{y}^{\prime}=\left[\begin{array}{lllll}
1+\tau_{1} \theta & 1+\tau_{2} \theta & 1+\tau_{3} \theta & \cdots & 1+\tau_{k} \theta
\end{array}\right]
\end{gathered}
$$

The matrix $\mathbf{H}$ can be illustrated as

$$
\mathbf{H}=\mathbf{D}+\mathbf{t} \mathbf{e}^{\prime}
$$

where

$$
\mathbf{D}=\left[\begin{array}{cccc}
a V_{1} & 0 & \cdots & 0 \\
0 & a V_{2} & \cdots & 0 \\
\vdots & \vdots & \ddots & \vdots \\
0 & 0 & \cdots & a V_{k}
\end{array}\right]
$$$$
\mathbf{t}^{\prime}=\left[\begin{array}{llll}
\tau_{1} & \tau_{2} & \cdots & \tau_{k}
\end{array}\right]
$$$$
\mathbf{e}^{\prime}=\left[\begin{array}{llll}
E_{1} & E_{2} & \cdots & E_{k}
\end{array}\right]
$$

The inverse of $\mathbf{H}$ is suggested in several textbooks of linear model such as Rencher [24] and Sen and Srivastava [25]. It yields

$$
\begin{aligned}
\mathbf{H}^{-1} & =\left(\mathbf{D}+\mathbf{t} \mathbf{e}^{\prime}\right)^{-1}=\mathbf{D}^{-1}-\frac{\left(\mathbf{D}^{-1} \mathbf{t}\right)\left(\mathbf{e}^{\prime} \mathbf{D}^{-1}\right)}{1+\mathbf{e}^{\prime} \mathbf{D}^{-1} \mathbf{t}} \\
\text { Therefore, } \mathbf{f} & =\mathbf{D}^{-1} \mathbf{y}-\frac{\left(\mathbf{D}^{-1} \mathbf{t}\right)\left(\mathbf{e}^{\prime} \mathbf{D}^{-1}\right)}{1+\mathbf{e}^{\prime} \mathbf{D}^{-1} \mathbf{t}} \mathbf{y}
\end{aligned}
$$

$$
\left.\mathbf{f}=\left[\begin{array}{c}
\frac{1}{a}\left[V_{1}^{-1}\left(1+\tau_{1} \theta\right)\right] \\
\frac{1}{a}\left[V_{2}^{-1}\left(1+\tau_{2} \theta\right)\right] \\
\vdots \\
\frac{1}{a}\left[V_{k}^{-1}\left(1+\tau_{k} \theta\right)\right]
\end{array}\right]-\left[\begin{array}{c}
\left(\frac{V_{1}^{-1} \tau_{1}}{\left(a+\sum_{m=1}^{k} \tau_{m} E_{m} V_{m}^{-1}\right)}\right)\left(\frac{\sum_{m=1}^{k} V_{m}^{-1} E_{m}\left(1+\tau_{m} \theta\right)}{a}\right) \\
\left(\frac{V_{2}^{-1} \tau_{2}}{\left(a+\sum_{m=1}^{k} \tau_{m} E_{m} V_{m}^{-1}\right)}\right) \\
\left(\frac{\sum_{m=1}^{k} V_{m}^{-1} E_{m}\left(1+\tau_{m} \theta\right)}{a}\right) \\
\left(\frac{V_{k}^{-1} \tau_{k}}{\left(a+\sum_{m=1}^{k} \tau_{m} E_{m} V_{m}^{-1}\right)}\right)
\end{array}\right)\right]
$$

Therefore, for the $j^{\text {th }}$ center, it yields

$$
\begin{aligned}
f_{c j} & =\left(\frac{V_{j}^{-1}\left(1+\tau_{j} \theta\right)}{a}\right) \\
& -\left(\frac{V_{j}^{-1} \tau_{j}}{\left(a+\sum_{m=1}^{k} \tau_{m} E_{m} V_{m}^{-1}\right)}\right)\left(\frac{\sum_{m=1}^{k} V_{m}^{-1} E_{m}\left(1+\tau_{m} \theta\right)}{a}\right)
\end{aligned}
$$

where $a=\sum_{j=1}^{k} \frac{1}{V_{j}}=\sum_{j=1}^{k} V_{j}^{-1}$,

$$
b=\sum_{j=1}^{k} \frac{E_{j}}{V_{j}}, \quad \tau_{j}=a E_{j}-b
$$

$$
\begin{gathered}
E_{j}=E\left(\hat{\theta}_{c j}\right)=E\left(\hat{p}_{c 1 j}-\hat{p}_{c 2 j}\right) \\
=\frac{n_{1 j} p_{1 j}+c_{1}}{n_{1 j}+2 c_{1}}-\frac{n_{2 j} p_{2 j}+c_{2}}{n_{2 j}+2 c_{2}} \\
V_{j}=V\left(\hat{\theta}_{c j}\right)=V\left(\hat{p}_{c 1 j}-\hat{p}_{c 2 j}\right) \\
=\frac{n_{1 j} p_{1 j}\left(1-p_{1 j}\right)}{\left(n_{1 j}+2 c_{1}\right)^{2}}+\frac{n_{2 j} p_{2 j}\left(1-p_{2 j}\right)}{\left(n_{2 j}+2 c_{2}\right)^{2}}
\end{gathered}
$$

In practice, we have to estimate the adjusted summary estimator by replacing the sample estimates for the unknown quantities: $E_{j}, V_{j}, p_{1 j}, p_{2 j}, \theta$. 